\title{
Pink Ocean Strategy: Democratizing Business Knowledge for Social Growth and Innovation
}

\author{
Evangelos Markopoulos ${ }^{1,2}$, Maria Barbara Ramonda ${ }^{3}$, Lisa Marie Carolin Winter ${ }^{4}$, \\ Haseena Al Katheeri ${ }^{5}$ and HannuVanharanta ${ }^{6,7}$ \\ ${ }^{1}$ University College London, School of Management, Gower St, Bloomsbury, WC1E 6BT \\ London, United Kingdom \\ ${ }^{2}$ Turku University of Applied Sciences. Faculty of Engineering and Business \\ Joukahaisenkatu 3, ICT-City, C-wing, FI-20520 Turku, Finland \\ ${ }^{3}$ Centro Motor Toyota SA, Av Colón 5077, X5000 Córdoba, Argentina \\ ${ }^{4}$ UVEX Winter Holding GmbH \& Co.KG, Wuerzburger Str. 181, 90766 Fuerth, Germany \\ ${ }^{5}$ Zayed University, College of Technological Innovation, P.O. Box 144534 Abu Dhabi, \\ U.A.E \\ ${ }^{6}$ University of Vaasa, School of Technology and Innovations, Wolffintie 34, 65200 \\ Vaasa, Finland \\ ${ }^{7}$ Poznań University of Technology, Plac Marii Skłodowskiej-Curie 5, 60-965, \\ Poznań, Poland \\ e.markopoulos@ucl.ac.uk,bramonda@centromotorsa.com.ar, lisa.winter@uvex.de, \\ Haseena.AlKatheeri@zu.ac.ae, hannu@vanharanta.fi
}

\begin{abstract}
In a constantly and rapidly changing world, innovation and organizational sustainability keep on being redefined. The integration of Blue and Green Ocean strategies can deliver social innovation and impact directly the lives of millions of people globally while assuring the needed organizational sustainability and profitability. This approach introduces the Pink Ocean Strategy concept, which cares about human life, integrity and dignity to booster innovation efforts. The new Pink Ocean Strategy is executed through the Company Democracy Model, the model for people, to embrace anyone who has and can share data, information, knowledge as well as wisdom creation for the good of the society, community and the humanity. The paper presents the six stages of the Company Democracy Model adjusted to navigate organizations into Pink Oceans, the overall strategic management framework, the pre and post conditions of each stage and the expected results per stage, enables this navigation to Pink Oceans.
\end{abstract}

Keywords: Pink Ocean · Green Ocean · Blue Ocean · Growth · Social · Sustainable Development Goals $\cdot$ Innovation · Democracy $\cdot$ Strategy $\cdot$ Management $\cdot$ UN - Shared Value $\cdot$ Social Innovation

\section{Introduction}

The continuous and rapid evolution of technology, as well as the global entrepreneurship dragged by the .com bubble and the start-up revolution, created a new entrepreneurial reality driven by new business ethics and values. The desire of the investors, to 
utilize bright young minds with innovative thinking, together with the dream and faith of the young entrepreneurs to succeed, in fame and wealth, as their start-up role models, shifted innovation directly to full capitalism where fast and profitable ideas dominated the attraction of interest, investors and funding.

This overall revolution created new markets where profitable innovation stands out of the competition in uncontested markets spaces as W. Chan Kim and Renee Mauborgne described it in the Blue Ocean Strategy [1]. Blue Oceans can be considered more of a metaphor or synonym to successful innovation and less than a strategy, as innovations that do not stand out in their market or outside of it, cannot be considered innovations. Therefore, innovation strategy is genuinely the Blue Ocean Strategy if innovation is effective and efficient. Building on that and following the significant global concerns related to global environmental and sustainable challenges, Evangelos Markopoulos, Ines Kirane, Clarissa Pipper and Hannu Vanharanta, extended the Blue Ocean Strategy into the Green Ocean Strategy for the development of innovative, sustainable and environmental technology-driven solutions in the sphere of Clean Tech [2].

The Green Ocean identifies the Blue Ocean's innovative elements and combines them with the knowledge needed to further innovate through the Company Democracy Model for democratic knowledge elicitation. The roadmap towards Green Oceans is purely strategic as decisions, dilemmas, risks, and opportunities need to be analyzed prior to entering the very niche green market. Furthermore, the Green Ocean Strategy comes in a time that is not only necessary but is also aligned with the maturity of the world on going green [3]. The public opinion towards a carbon-free word and environmentally sustained is stronger than ever. This situation strengthens innovations that in other periods could have been questioned much on their business sustainability, originality, profitability, and scalability. However, there are significant societal issues that are not being addressed with the capitalistic Blue Ocean Strategy and the environmentalist Green Ocean Strategy. Above the creation of wealth and a carbon-free world, it is essential to help people live their lives decently in order to enjoy the carbon-free world with economic prosperity. Most of the 17 United Nations Sustainable Development Goals are directly related to the efforts towards the development of social behavior to address the significant human needs. These goals, among the rest, have care, love, and support as a common nominator to achieve and sustain human dignity above all.

\section{Innovation Evolution}

The term 'Innovation' is constantly identified as a fundamental phenomenon of social and economic business advancements [4]. Firms advocate for innovation and its ability to develop their standing, ideally respective to their competitors, within their market. The conversion of ideas to the invention and their commercialization in products or services creates value for their customers and shareholders. Innovations, however, should be replicable at an economical cost that can satisfy a specific corporate need and target group. This process comprises the intentional application of information, imagination, and initiative to derive values from established resources to spawn or adapt useful products or services [5]. 
Innovation can be primarily categorized into Radical, Incremental, and Lateral Innovation areas. In Radical Innovation inventions eradicate existing business models, exterminate existing systems or processes and substitutes them with something completely new. Incremental Innovation improves existing systems, processes, methods, products or services, while Lateral Innovation replicates existing innovations in the industry.

Within these categories, different types of innovation exist, focusing on Technology, Processes, Business Models, Positioning, and others, ranging from Disruptive Innovation to Frugal Innovation to Service Innovation or Shared Value Innovation. Figure 1 presents a classification of the innovations types with the three main categories.

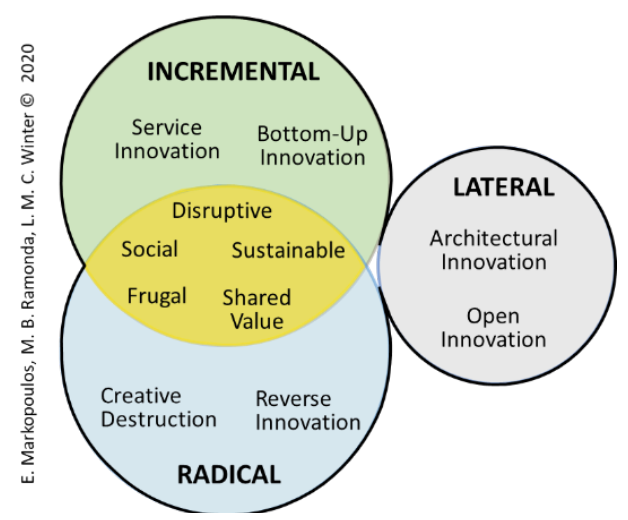

Fig. 1. Innovation Categorization

In brief, Disruptive Innovation builds unique and new markets and reshapes existing ones [6] while Frugal Innovation discovers new business models, reconfigures value chains, supplies functional solutions with fewer resources and redesigns products towards affordability scalable and sustainable manners [7]. Bottom-Up Innovation focuses on implementing inventions by employees, in contrast to top-down innovation [8]. Social Innovation targets social needs through the implementation of new strategies and active collaboration with constitutions across governments, businesses and the nonprofit world [9]. Open Innovation uses multiple external sources to drive innovation instead of relying on their internal knowledge and resources. Reverse Innovation applied to the inventions and production of ideas, products or services in emerging economies prior to their commercialization in developed economies [10]. Service Innovation changes the processes of how customers are served to increase value, while Architectural Innovation takes technologies, processes or ideas and implementing them into new markets. Sustainable Innovation implements sustainable development solutions for the environment, society, and the economy. Lastly, Shared Value Innovation meets societal needs profitably, by leveraging resources and innovations from the private sector; creating new solutions for society's most demanding problems [11]. 


\section{Innovation Oceans. From the Red to the Blue, to the Green.}

In 2004 the Blue Ocean Strategy revolutionized the business sector by urging organizations to set aside competition between them and give room to the expansion of markets through innovative ideas [1]. The term Red Ocean was an invention to describe the saturated 'bloody' market-driven competition in existing market spaces, continue exploitation for demand on the same target, value-cost trade-off groups and price differentiation. The majority of the organizations today continue to compete defined and predictable markets, with known competitors and with traditional management structure. The fear of the unknown, the cost of innovation, and the long term results in many cases keep organizations in Red Oceans to keep on trying to make the most of the existing markets [1]. Alternatively, Blue Oceans mobilize companies to generate new market spaces and make the competition irrelevant. Thus, it creates and captures new demand through innovation, breaks the value-cost trade-off and aligns the activities of the organization towards differentiating and low cost. It is those who thrive to create innovative ideas that will find opportunities for rapid and profitable growth [1].

Recently, Prof. Evangelos Markopoulos and Ines Selma Kirane created an extension of the Blue Ocean theory, by driving innovations towards environmental sustainability with Green Ocean Strategy. The Green Ocean is an evolution of the Blue Ocean by capturing new social markets and targeting shared value competitiveness. Its essence is of a democratic driven culture that uses its human intellectual capital to develop environmentally sustainable and economic innovation by the society for the society [2].

Attempts have been made in the past to develop other oceans emphasizing strategic alliances towards going Blue but did not seem to have the expected acceptance. An example of this is the Purple Ocean Strategy by Dr. Kittichok Nithisathian and Prof. John Walsh, which integrated the Red and Blue Oceans, based on the logic that Blue Oceans do not guarantee long term organizational success and sooner or later they will turn Red again. Furthermore, it considers organizational operations is both the contested and uncontested markets and suggests beating the competition by focusing on the customer and emphasizing the value of "free", zero cost [12]. The Purple Ocean was positioned between the Red and the Blue Oceans, indicating that it is wise to stand in the middle without being conservative and compete in a saturated market but also without seeking the new big idea.

The constant development of new strategies however, under the concept of the 'ocean' as new market space, highlights an innovation gap that can be fulfilled with more specialized and targeted strategies.

\section{$4 \quad$ Mind the innovation gap}

The emergence of entrepreneurs and innovators who intend to combine financial sustainability with social impact is a great novelty. There are many types of social enterprise but the essential aspect is based on having pure intentionality for change that is more powerful and impactful than profit.

While it is true that the engine driving social innovation is made up of collaboration, empathy, sensitivity to dilemmas, as well as the desire to strengthen the environment of societies, this entrepreneurial passion does not seem to be enough. It is important for 
them to utilize the best any strategy and model available regarding social innovation for their efforts to be sustainable and simultaneously philanthropic.

Despite the evolution of the various Ocean Strategies, from Red to Blue and Blue to Green, innovation continues to grow rapidly. Therefore, there is yet a need for a strategy to covers socio-economic trends and needs.

The Blue Ocean Strategy focuses on finding unexplored markets through innovation, whilst the Green Ocean Strategy focuses on extending innovation towards environmental sustainability and viability. By observing the evolution of the innovation oceans, it seems that one ocean comes to complete the other.

The Red Ocean Strategy created a gap of innovation that was covered by the Blue Ocean with its pure capitalist nature but created a gap of environmental and sustainability innovation as Green markets were smaller with more expensive products and demanding costumes. The Green Ocean Strategy covered this gap by utilizing the global environmental movement and customer maturity. However, not all needs have been covered and it is not possible to be covered by only one strategy. The need for humanitarian innovations is a major one. Blue Ocean does not address it directly and Green Oceans leans on it satisfying the environmental dimension. This innovation gap indicates that traditional approaches must be complemented by the exploration of change, and new approaches need constant modernization.

\section{Social Innovation Challenge}

In a world where the expansion of wealth goes above all for many, and the battle for a carbon-free world is taking over, the value of people's dignity must not be forgotten. This social dimension must be supported by providing people help to live their lives honorably and satisfactorily in order for them to savor the Green carbon-free world with the Blue economic prosperity.

The 2030 Agenda for Sustainable Development which was adopted by all the United Nations Member States in 2015 proposed 17 Sustainable Development Goals (SDGs). The goals provide a blueprint for peace and prosperity for people and the planet, today and in the future. SDGs embody a crucial call for action by all countries in a global partnership. They honor that ending poverty and other deprivations go in close association with strategies that can improve health and education, reduce inequalities and drive economic growth, all while confronting climate change and working to preserve life on land and below water [13].

Most of the 17 SDGs such as goal 1 (No Poverty), 2 (Zero Hunger), 3 (Good Health and Well-Being), 4 (Quality Education), 5 (Gender Equality), 6 (Clean Water and Sanitation), 8 (Decent Work and Economic Growth), 10 (Reduce Inequalities), 11 (Sustainable Cities and Communities), and 17 (Partnerships), align the efforts towards developing social behaviour to address the major human needs of the society [14].

The common denominator characteristics of the UN SDGs are care, love, and support, which are needed to achieve and sustain human dignity above all. It is vital to focus on complying with these SDGs to achieve prosperity in the business world, and reach economic growth while protecting the environment and empowering societies. 


\section{The Pink Ocean Strategy - Thinking beyond profit}

The process of integrating the UN SDGs in a strategy interlinked with the current Blue and Green Ocean strategy leads to the creation of the Pink Ocean Strategy (Fig. 2). The Pink Ocean Strategy can directly impact the lives of millions globally while at the same time assuring the needed sustainability and viability in the environment and society, with profitability growth and scale-out business operations development.

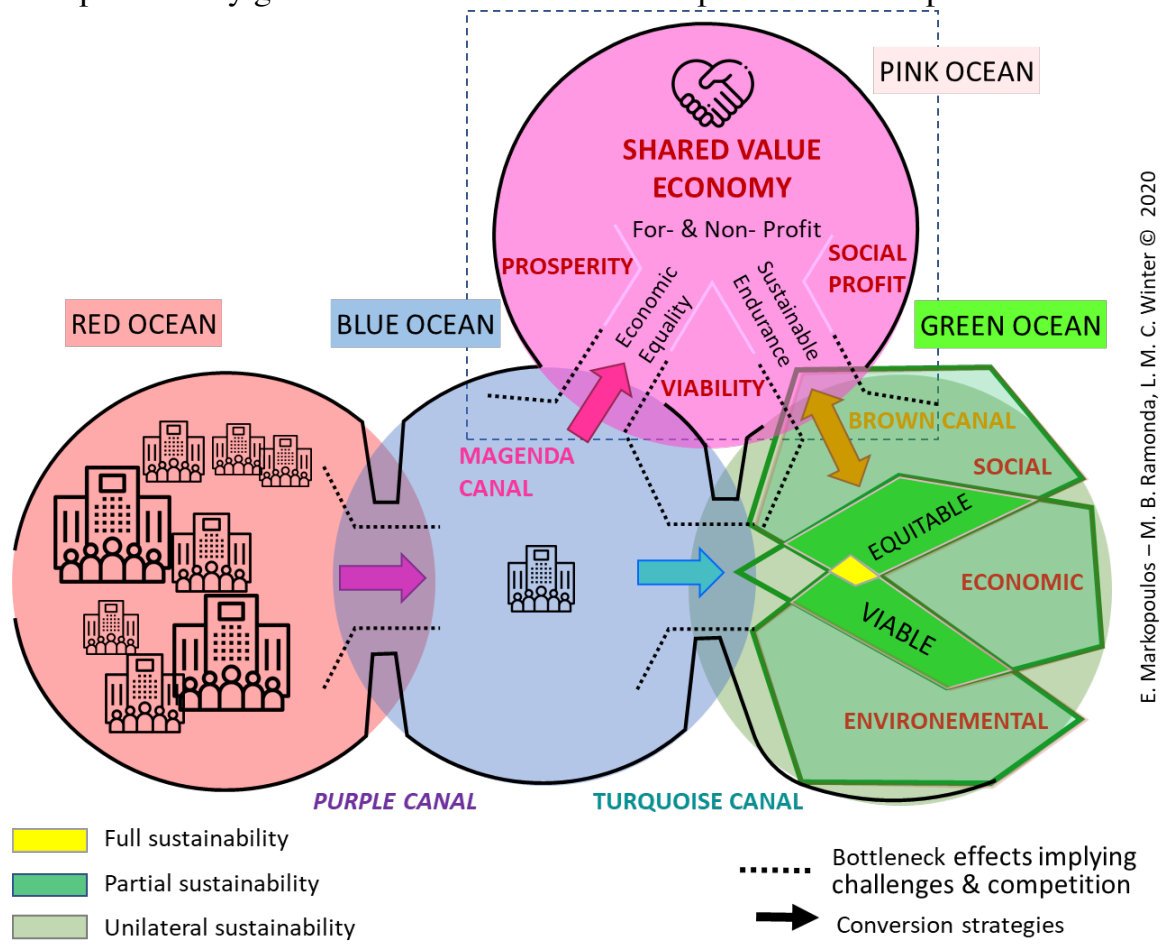

Fig. 2. The Pink Ocean Strategy

The Pink Ocean Strategy is introduced at a time the world seeks new alternatives of innovative thinking to rising problems such as climate change and inequality. The old paradigms on the paternalistic assistance of the state or the companies to address these problems are, indeed, long gone. In fact, the world has evidenced a revaluation of the role of innovation in society and the enormous impact it can have on the sustainable growth of nations. Therefore, in order to contribute to the development of existing innovation tools the Pink Ocean Strategy aims to create a space for growth which allows contribution of ideas towards the construction of a social innovation ecosystem, but also an environments to test ideas, approaches, dynamics and models, that are likely to become useful solutions to global and regional social concerns.

To navigate to the Pink Ocean, an organization has to go through the Blue Ocean since innovation remains a precondition for progress beyond the competition. Achieving social innovation requires innovation, and innovation is achieved through Blue Oceans. 
However, the thinking of utilizing an innovative idea, process or product is what differentiates the path an innovation can follow or stay into the pure capitalism and profit-driven Blue Ocean. There is nothing wrong with innovations to stay in Blue Ocean as this is a significant achievement on its own, but extending beyond the Blue, there must be a drive for it and a fair market for the innovation to be sustainable.

The innovation transition process from Blue to Green or Pink is achieved through a process canal. Green Ocean is reached through the turquoise canal, a process that transforms innovation into environmental social and sustainable. Pink Ocean, however, is reached through the magenta canal, a path that emphasizes more into sustainability by sharing and caring for the innovation benefits. It is the economic equality that leads organizations from the Blue to the Pink Ocean where a shared value-driven culture to sustain human dignity overrules any other success goal.

Reaching Pink Oceans opens the pathways to extent into Green as both oceans are characterized by social and sustainable values and goals. The success an innovation can reach in social communities and assure sustainable endurance can extend the social good and contribution to the society by transforming parts of its operations or the entire innovation to address a more sustainable goal, enlarging this way its target group.

\section{$7 \quad$ Pink Ocean Strategy Pathways}

Organizations can implement Pink Ocean Strategy either through various paths connected with the Blue and Green Ocean Strategies (Fig.3). The main characteristics of the Pink Ocean Strategy are compassion and care driven for those who need help, opportunity and an environment to create, develop and prosper. Achieving prosperity by offering to people motivation, fairness, and care, it is certain that success will return sooner or later to the investments made on these people. 


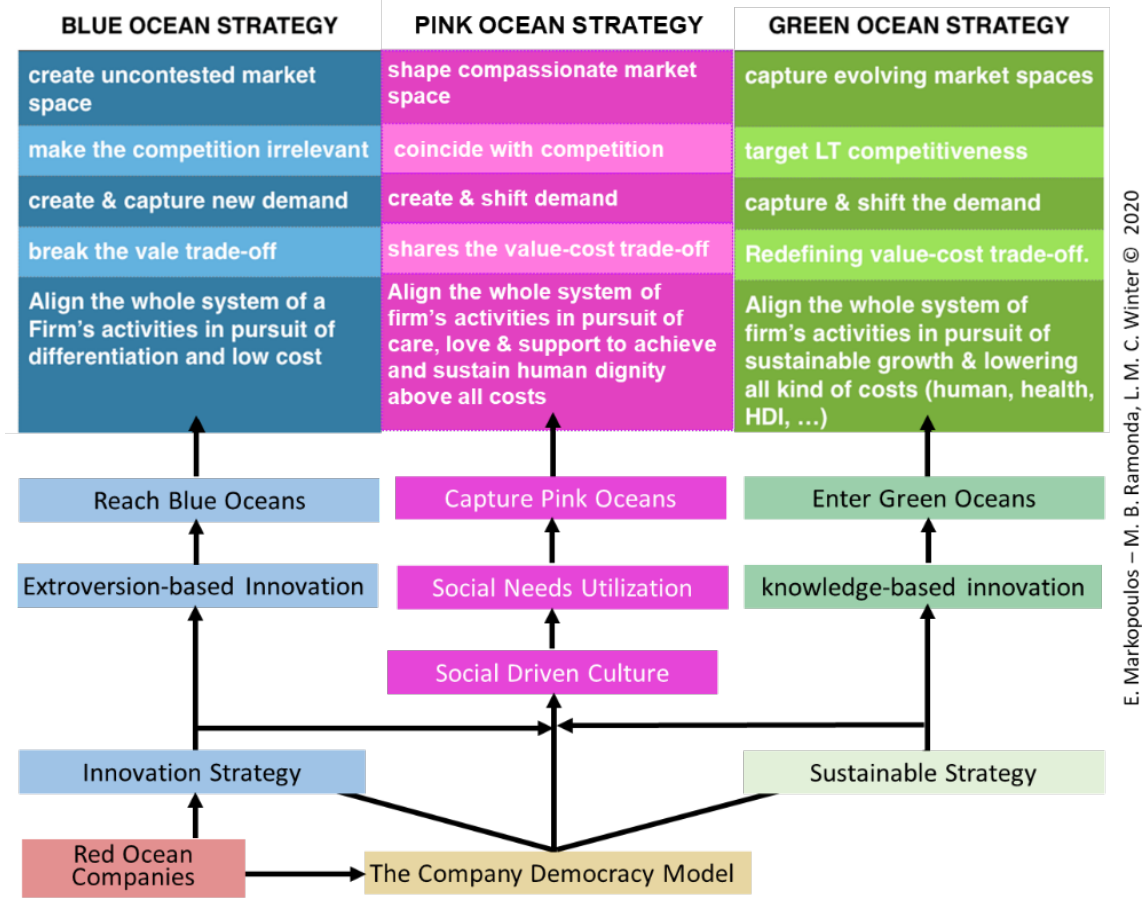

Fig. 3. Pink Ocean Strategies classifications \& paths for achievement

The Pink Ocean shapes a compassionate market space and not competitive. It is a long term people based and not profit based investment. The Pink Ocean coincides with the competition as opportunities are offered to those who seek them. This approach creates and shifts the demand for opportunities to the less privileged ones. As a shared value strategy, Pink Ocean shares the value-cost trade-off and aligns the overall system of the firm's activities in pursuit of care and support towards achieving and sustaining human dignity above all costs.

Unlike Red Oceans, the Pink Ocean requires a democratic organizational culture that can enable shared and care value thinking and support innovation efforts based on that. It is for certain that not all organizations, and even more not all people feel or believe that share and care value innovations can achieve a return on investments. To tackle this issue employees in an organization must democratically be selected, and not obliged, to think and work towards Pink Ocean innovations.

Corporate democratization can either be the base for the organizational innovation strategies, or derive from the Red Ocean strategy as an alternative part to the more expected, accepted, and profitable Blue Oceans. The same path can be followed for Green Ocean strategies as both Pink and Green Oceans differentiate the profitability strategy form the Blue Oceans which is the next and logical evolution. 


\section{$8 \quad$ The Pink Ocean Strategy Processes}

The element of democratization in an organizational culture is crucial for reaching Pink Oceans. Share and care value cannot be achieved by imposing and mandating people to share or care. It can only be possible if those who do care and share are identified in the company and supports by turning their ideas into effective innovations in products and services. The Company Democracy Model by Prof. Evangelos Markopoulos and Prof. Hannu Vanharanta forms the base on which the Pink Oceans process evolves. The mode is structured into an evolutionary six-level pyramid in which knowledge is identified at level 1 and transformed into a global competitive advantage in level 6 [15]. The Company Democracy Model has been primarily developed for the creation of Blue Oceans but over time variations of it have been created to address various organizational goals and needs, with one of them to be the creation of shared value. This challenge has been approach by emphasizing on the identification of the human intellectual capital and the progression of the individual, and not only the idea or the innovation, over the six evolutionary levels, with the innovation benefits to be shared between the employee and the organization [16]

The six levels of the Pink Ocean strategy start with the identifications of the share and care value thinkers and ends with the institutionalization of the innovation and its adaptation on a global scale (Fig. 4).

Level 1 on the Pink Ocean Strategy implementation model deals with the creation of an organizational culture open to share and care value ideas, knowledge and contributions. It is the level that allows all who feel and need to share their knowledge for a common good, to express themselves and propose their solutions.

Level 2 is based on the creations of teams between those who have shared value ideas with the experts needed to implement them, but also with external parties such as NGOs and social organizations related to the innovation concept and needed to provide their experience and expertise.

Level 3 is based on the implementation of the shared value idea into a working prototype that can be tested by the NGO or the social organization that joined the team at level 2 . This is the level where the actual knowledge and idea is practically implemented into a product or service and tested as well.

Level 4 extends the implementation of the innovation achieved in level 3 by rolling it out to the members and user of a community to identify areas of improvement, usage concerns, receive valuable feedback and actually let the innovation mature for some time while the users/clients enjoy the innovation services with limited charges or free.

Level 5 measures the success of level 4 and identifies the most competitive and rewarding elements of the innovation that can be monetized for the innovation to generate the needed income to maintain operations and build on it. The selection and the monetization of such elements are related to the value they return to the user/client.

Level 6 moves the successful innovation (from level 4), and sustainable as well (from level 5) to comply with international sustainable goals, standards, protocols, and treaties to become institutionalized and roll out its application and distribution globally. This is a double target/benefit level. The institutionalization of the innovation can help its financial sustainability but also its recognition and adaptations by various organizations, NGOs and governments worldwide, which will support it financially as well. 
It must be noted that the colors of the pyramid change from pink to blue and back to pink again as the process goes through a stage where innovation monetization practices are applied to the innovation competitive features in order to obtain financial sustainability needed for the innovation to continue services the people. These practices follow more of a Blue thinking logic to assure suitability than the Pink thinking caring logic.

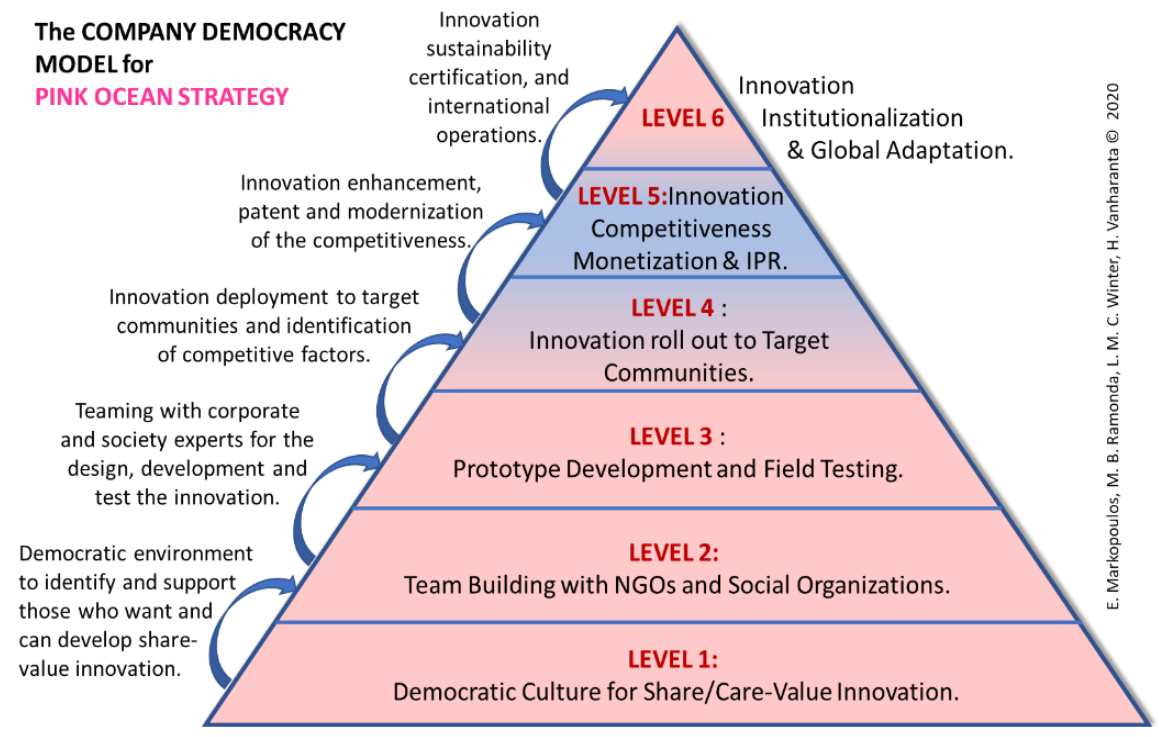

Fig. 4. The Pink Ocean Strategy Levels

\section{Implementing the Pink Ocean Strategy}

The implementation of the Pink Ocean Strategy is archived by the effectiveness an organization moves in the magenta canal. The canal, whose color is the combination of blue and pink is the transition process from the needed Blue Oceans Strategy to the desired Pink Ocean Strategy. It is the execution path of the Pink Ocean Strategy levels. To archive, this transition several preconditions and post-condition much be satisfied but also the timing the organizations decides to go Pink (Fig. 5) 


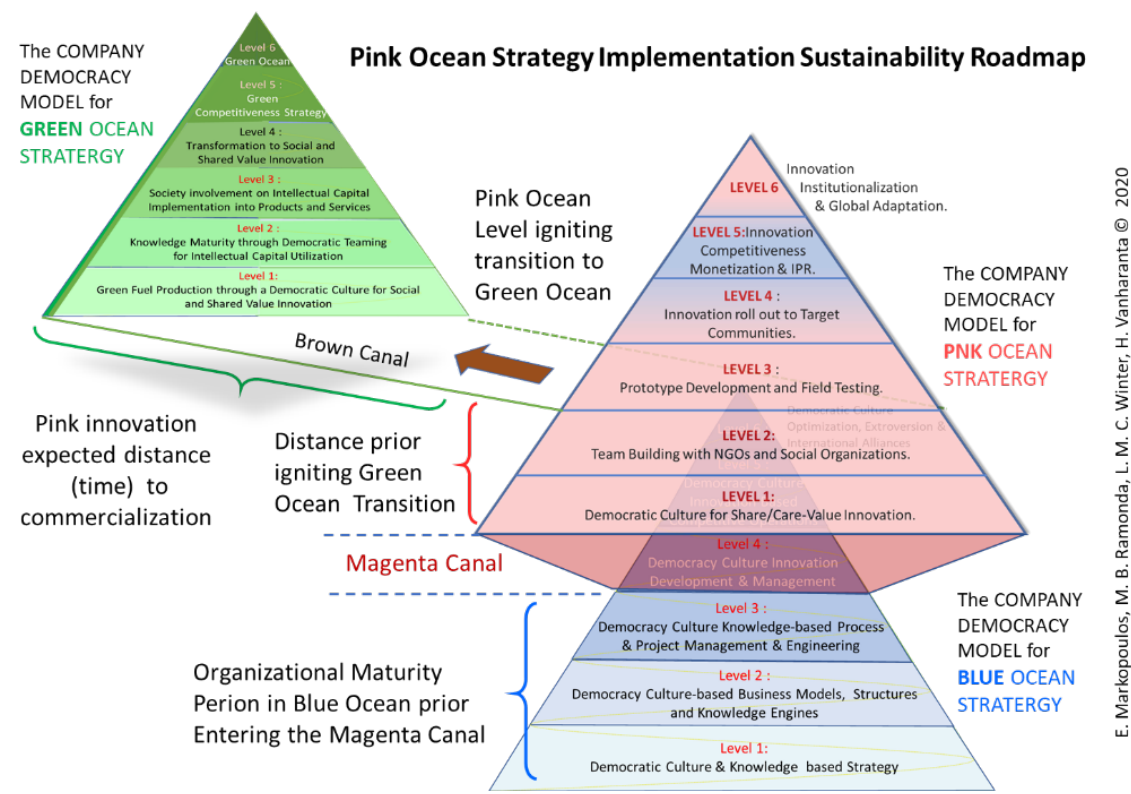

Fig. 5. Pink Ocean Strategy Implementation and Sustainability Roadmap

The preconditions are related primarily to the needed innovation culture the organization shall have. Regardless of the type of innovation or the innovations strategy, the organization must empower innovative thinking, promote collectiveness, and reward contributions that improve its operations either as simple processes or advanced innovations on revolutionary products and services. Such preconditions can be achieved with the adaptation of the Company Democracy Model prior to moving into the Pink Ocean Strategy. This can also be an alternative path for the organizations to reach the needed Blue Oceans required to ignite the initiative towards Pink Oceans.

The pre-condition of Blue Oceans is important for one more reason which is sustainable for the Pink Ocean initiatives until they become sustainable themselves. Pink Ocean has driven initiatives to achieve financial sustainability at levels 4 or 5. This means that in more than three-quarters of the Pink Ocean process, all the effort is being funded only by the organization itself, without any revenue.

It is therefore recommended for an organization to enter the magenta canal once the Blue Oceans have profitability excess. This is usually achieved when the organization reaches level 4 in the Company Democracy Model, meaning that the Blue Ocean innovation passed level 3, which is the market accept level, and it is at global operations by utilizing its competitive advantages. However, financially stable organizations can enter the magenta canal at the early stages of the Blue Ocean journey (level 2 or 3 of the Company Democracy Model).

The postconditions of the Pink Ocean Strategy Implementation is its financial sustainability. Shared value and social innovation can lack enough financial sustainability even if they can massively attract and sustain innovation users, followers or clients in the best case. Therefore, it is important for the innovation to extend into spaces where similar social concerns are shared, where the population of these innovation areas is 
more keen pay, maintain subscriptions or become loyal to the offered products. One of these spaces can be environmental sustainability which also touches upon several UN SDGs that form the target groups of the Pink Ocean. Therefore the transition from Pink to Green in either selective innovation elements holistically is a postcondition to be determined by the degree of the innovation success along the Pink Ocean.

\section{Areas of further research}

The Pink Ocean Strategy model presented in this paper is a first attempt to model a new innovation-based organizational management strategy. The model is based on the concept of organizational democratic cultures which makes sense but its practically not easy to be achieved even by organizations proud of their democratic processes.

In order for the model to gain more strength and become more promising on the results excepted to deliver, it is important to develop a number of metrics and indexes that can monitor the progress of the Pink Ocean Strategy execution and the benefits gained by the organizations through them. Of courses, this metrics-driven execution process contradicts the ideas that Pink Oceans are generated to bring innovation to the ones in need and therefore performance measurements cannot be applied in this case. This valid statement is true but it is also true that Pink Oceans achieve financial sustainability late, as well are late return on investment. Until then the organizations need to track any kind of progress translated into any kind of benefits which does not need to be financial. This set of metrics that will extend this work emphasizes the identification of various benefits that directly or indirectly will positively impact the organizations to continue seeking Pink Oceans. Such benefits can be related to the time an organization stays in the magenta canal, to employee satisfaction and loyalty, to the impact of the Pink Ocean activities on the organizational reputation on ethical governance, to social corporate responsibility, to sustainability with the UN SDGs and others targets. The sum of these measurable impacts will provide the Pink Ocean Strategy a quantified progress picture and the support needed to reach its goal.

\section{Conclusions}

According to Heraclitus 'change is constant'. The evolution of management, especially the last fifty years with the internationalization and the globalization eras, has moved into multidisciplinary dimensions through which organizations constantly seek the transformation that will not only progress them but above all will sustain them.

The Pink Ocean Strategy presented in this paper provides a new strategic management and leadership thinking organizations can explore building reputation benefits besides the financial ones. The UN Sustainable Development Goals were created to provide help and relief to the people in need and the planet, but at the same time, they opened massive target market sensitive to the societies, the SDG movement and the people as well. Approaching effectively this new distribution of priorities is as effective as approaching new target groups aligned with the pubic feeling and concerns.

The Pink Ocean Strategy aims to extend the term of innovation from the old meaning served by the Blue Ocean concept to a new social meaning. It is a strategy that places 
the humans and the societies first and above any profitable target. Profitability will be achieved after all by the determination organizations have to truly sail on Pink Oceans.

\section{References}

1. Kim, W.C., Mauborgne, R.A.: Blue Ocean Strategy: How to Create Uncontested Market Space and Make the Competition Irrelevant. Harvard Business Review Press, Massachusetts (2015)

2. Markopoulos, E., Kirane, I.S., Piper, C., Vanharanta, H.: Green Ocean Strategy: Democratizing Business Knowledge for Sustainable Growth. In: Ahram, T., Karwowski, T., Taiar, W. (eds.) IHSED 2019. AISC 1026, pp. 115--125. Springer Nature Switzerland AG, Basel (2020)

3. Jacobs M. : Green Growth: Economic Theory and Political Discourse. Grantham Research Institute on Climate Change and the Environment. Working papers 108 and 92 (2012)

4. Andersen, E.S., Martin, B.R., Fagerberg, J.: Innovation Studies: Evolution and Future Challenges. Oxford University Press, Oxford (2013)

5. Yaacob, A.S., Fazal, S.A., Kumar, N., Mohd Zawawi, N.F., Al Mamun, A.: Defining the Concept of Innovation and Firm Innovativeness: A Critical Analysis from Resource-Based View Perspective. In: 11th International Journal of Business and Management, pp. 87--94. Canadian Center of Science and Education (2016)

6. Harvard Business Review, https://hbr.org/2008/10/how-ti-spot-disruptive-innovat

7. Sammut-Bonnici, T., McGee, J.: Frugal Innovation. In: 12th Wiley Blackwell Encyclopedia of Management of Strategic Management. John Wiley and Sons, Ltd., Hoboken (2014)

8. Park, S., Kim, J.N., Krishna, A.: Bottom-Up Building of an Innovative Organization: Motivating Employee Intrapreneurship and Scouting and Their Strategic Value. Management Communication Quarterly, pp. 1--30. Sage, West Lafayette (2014)

9. Stanford Graduate School of Business, https://www.gsb.stanford.edu/faculty-research/centersinitiatives/csi/defining-social-innovation

10. Seke Mboungou Mouyabi, J.: Reverse Innovation: A Revolution of the Present and Future of the Emerging Markets. International Journal of Engineering Research and Management 04, pp. 6--14 (2017)

11. Porter, M.E., Kramer, M.R.: Creating Shared Value. Harvard Business Review (2011)

12. Nithisathian, K., Walsh, J.C.: Purple Ocean Strategy Concept Paper: Content Analysis from Interviews and Literature Review. SIU International Conference, Bangkok (2013)

13. Sustainable Development Goals, https://sustainabledevelopment.un.org/?menu=1300

14. About the Sustainable Development Goals, https://www.un.org/sustainabledevelopment/sustainable-development-goals/

15. Markopoulos E., Vanharanta H.: Democratic Culture Paradigm for Organizational Management and Leadership Strategies - The Company Democracy Model. In : Proceedings of the 5th International Conference on Applied Human Factors and Ergonomics. Poland (2014)

16. Markopoulos E, Vanharanta H.: Company Democracy Model for Development of Shared Value, Elsevier, Procedia Manufacturing, Volume 3, pp 603--610 (2015) 